\title{
Sap flow measurement by a single thermal dissipation probe: exploring the transient regime
}

\author{
Imen MAHJoub $^{1}$, Mohamed M. MASMoudi ${ }^{1 *},{\text { Jean P. } \text { LHOMME}^{2} \text {, Netij BeN MeChliA }}^{1}$ \\ ${ }^{1}$ INAT, 43 Avenue Charles Nicolle, 1082 Tunis-Mahrajène, Tunisie \\ ${ }^{2}$ IRD (UR 060), CEFE-CNRS, 1919 route de Mende, 34293 Montpellier, France
}

(Received 4 November 2008; accepted 28 February 2009)

Keywords:

sap flow /

thermal dissipation /

single probe /

transient regime /

olive species

\begin{abstract}
- In thermal dissipation method the stationary regime is used to estimate sap flow density from the temperature difference between a heating probe and a non-heating one.

- A new approach, based on the non-stationary regime of a single heating probe, is investigated on an olive tree branch. Probe temperature is monitored for different flow densities and for heating and cooling modes. Measurement sequences of $10 \mathrm{~min}$ using $5 \mathrm{~s}$ time steps are made just after the heating is switched on or off.

- Results show that temperature variation has a quasi-exponential phase whose duration decreases for increasing flow densities. Thermal indices using temperature of the probe measured at initial, final, and intermediate times $t_{i}$ were developed. Relationships between sap flow density and thermal indices were found to be linear for $t_{i}=10 \mathrm{~s}-60 \mathrm{~s}$ with better coefficients of determination in cooling mode $\left(R^{2}=0.94-0.96\right)$ than in heating $\left(R^{2}=0.83-0.93\right)$. Validation tests confirmed the robustness of the single probe method with lowest error obtained for $t_{i}=20 \mathrm{~s}$.

- Single probe method seems to be a promising technique to measure tree sap flow. Its application could be considered under cooling mode using $t_{i}=20 \mathrm{~s}$ and relatively short measurement sequences e.g. 5-10 $\mathrm{min}$.
\end{abstract}

\author{
Mots-clés : \\ flux de sève / \\ dissipation de chaleur / \\ sonde unique / \\ régime transitoire / \\ olivier
}

\begin{abstract}
Résumé - Mesure du flux de sève par sonde de dissipation thermique unique : exploitation du régime transitoire.

- La méthode de dissipation de chaleur utilise le régime stationnaire pour estimer la densité de flux de sève à partir de la température d'une sonde chauffée et une sonde non chauffée.

- Une nouvelle approche exploitant le régime transitoire d'une seule sonde est testée sur une branche d'olivier. Des séquences de mesure de la température de la sonde sont réalisées pour différentes densités de flux. Les mesures sont effectuées toutes les $5 \mathrm{~s}$ pendant les 10 min qui suivent la mise en route ou l'arrêt du chauffage.

- La variation de la température présente une phase quasi-exponentielle de durée inversement proportionnelle à la densité de flux. Des indices thermiques utilisant les températures de la sonde aux instants initial, final et intermédiaire $t_{i}$ ont été développés. Les relations entre les densités de flux et ces indices sont de type linéaire pour $t_{i}=10 \mathrm{~s}-60 \mathrm{~s}$ avec des coefficients de détermination plus élevés en refroidissement $\left(R^{2}=0.94-0.96\right)$ qu'en chauffage $\left(R^{2}=0.83-0.93\right)$. Un travail de validation a confirmé la robustesse de la méthode, l'optimum étant $t_{i}=20 \mathrm{~s}$.

- La méthode de la sonde unique semble être une technique prometteuse pour mesurer le flux de sève des arbres. Son application est envisageable en mode refroidissement avec $t_{i}=20 \mathrm{~s}$ et des séquences de mesure relativement courtes : 5-10 min.
\end{abstract}

\section{INTRODUCTION}

Sap flow measurement is an important technique for studying in situ plant-water relations. The method originally devised

\footnotetext{
* Corresponding author:

masmoudi.med@inat.agrinet.tn/netij.benmechlia@iresa.agrinet.tn
}

by Granier (1985; 1987), based on a thermal dissipation probe, is one of the most frequently used. The probe consists of two cylindrical needles inserted radially into the stem. One needle, placed above the other, contains a heating element, supplied at constant power by Joule effect. The heating needle exchanges sensible heat with its environment made of wood and sap. The 
physical process responsible for the transfer of heat from the needle to the wood involves both conduction in a solid medium (xylem vessels) and convection in a fluid in motion (sap flow). In stationary regime, a relationship linking sap flow density with the temperature difference between the probe needles can be derived and constitutes the basis of Granier's method of sap flow measurement. This relationship apparently does not depend on tree species when sensors geometry (e.g. effective length) and heating power remain the same, which was confirmed by other studies (Lu et al., 2004). The sap flow for the whole plant is obtained by multiplying the sap flow density by the sapwood area at the point of measurement, which is not always easy to determine accurately. Due to its simplicity, low cost and reliability, Granier's stationary method has been widely used, particularly in forestry (Andrade et al., 1998; Braun and Schmid, 1999; Lüttschwager and Remus, 2007; Ma et al., 2008; Masmoudi et al., 2004; Meinzer et al., 1999; Oliveras and Llorens, 2001; Sevanto et al., 2008; Wilson et al., 2001; Wullschleger and Hanson, 2008). However, substantial errors on sap flow estimates are induced by heat storage, especially in small stems, and by natural thermal gradients in the wood (Cabibel and Do, 1991; Do and Rocheteau, 2001a). Additionally, the zero flow conditions needed for sap flow density calculation (often observed at predawn) may not occur under certain circumstances, such as during the slow restoration of internal tree water storage following a prolonged drought period (Lu et al., 2004).

Do and Rocheteau (2002b) use a cyclic heating instead of the continuous heating in order to correct sap flow estimates for natural thermal gradients. They measure probe temperature difference at the end of heating periods and subtract from it the probe temperature difference at the end of cooling periods. When cyclic heating is used, the stationary regime does not occur except for high flow densities, which requires a specific calibration to account for the non-stationary regime. The cyclic heating substantially improves the accuracy of sap flow measurement in situations where thermal gradients are important (Lu et al., 2004). Yet, the determination of zero flow conditions is still required. Do and Rocheteau (2002b) pointed out that the measurement principle of the cyclic heating would apply to a single heating needle probe. Our work follows this statement, but it analyses the single probe temperature occurring just after current is switched on or off, in order to develop the basis for a new approach of measurement. Specifically, it explores the pattern of temperature variation during the transient regime and how it is correlated to flow density.

\section{MATERIALS AND METHODS}

\subsection{Theoretical basis}

When the thermal dissipation probe is being heated by Joule effect through a given resistance and with a given current intensity, the temperature of the probe reaches a steady-state regime characterized by an equilibrium temperature. The theoretical basis of this stationary regime was first developed by Granier (1985) and then completed by
Valancogne and Granier (1991). In this theory the heat flux $\varphi$ transferred from the probe to the wood is written as

$$
\varphi=h(u)\left(T-T_{w}\right)
$$

where $T$ is the temperature of the probe, $T_{w}$ is the average temperature of the wood around the probe (the temperature field is assumed to be homogeneous) and $h$ is the exchange coefficient, expressed as a function of the flow density $u$ as

$$
h(u)=h_{0}\left(1+\alpha u^{\beta}\right)
$$

where $\alpha$ and $\beta$ are two empirical coefficients ( $\beta$ is assumed to be equal to 1 in Granier's original paper) and $h_{0}$ is the value of the exchange coefficient at zero density (hence, representing the conductive part of the heat transfer).

When heating current is switched off, the heat lost by the probe is dissipated within the medium and its temperature $T$ decreases more or less rapidly down to the temperature of the wood, satisfying the transient heat balance equation written in the form of the following differential equation

$$
C \frac{d T}{d t}+h(u)\left(T-T_{w}\right)=0
$$

where $C$ is the heat capacity of the probe and $t$ is time. Assuming $T_{w}$ to be constant during the integration time, the solution of this firstorder linear differential equation is

$$
T(t)-T_{w}=\left(T_{0}-T_{w}\right) \exp \left[-\int_{0}^{t} h(u) d t / C\right]
$$

where $T_{0}$ is the temperature of the probe at the initial time of the kinetic ( $t=0$, when the current is switched off). The temperature of the wood $T_{w}$ represents the probe temperature at equilibrium (when $t \rightarrow+\infty$ ). According to this theory, probe temperature $T$ follows a pseudo-exponential decay with time, which depends upon the heat capacity of the probe $C$ and the coefficient of heat exchange between the probe and the wood $h(u)$. If $u$ is assumed to be constant during the integration time, the coefficient of the exponential reduces to $-h(u) t / C$ and the decay becomes strictly exponential with time. Inverting equation (4) leads to the following expression

$$
y(t)=\ln \left(\frac{T_{0}-T_{w}}{T(t)-T_{w}}\right)=\frac{1}{C} \int_{0}^{t} h(u) d t .
$$

Taking into account equation (2) with $\beta=1$, the integral in equation (5) can be expressed as a function of the mean sap flow density $\bar{u}$ (between 0 and $t$ )

$$
\int_{0}^{t} h(u) d t=h_{0}(1+\alpha \bar{u}) t
$$

This means that for a given value of flow density, the function $y(t)$ is a straight line. Its slope varies with flow density: the greater the flow density, the higher the slope of the line. For any intermediate time $t_{i}$ an index $I$ is defined as

$$
I\left(t_{i}\right)=\frac{1}{t_{i}} \ln \left(\frac{T_{0}-T_{w}}{T\left(t_{i}\right)-T_{w}}\right)
$$

where $T\left(t_{i}\right)$ is the temperature of the probe at $t=t_{i}$. Equations (5) and (6) show that there is a one to one relationship between the mean 


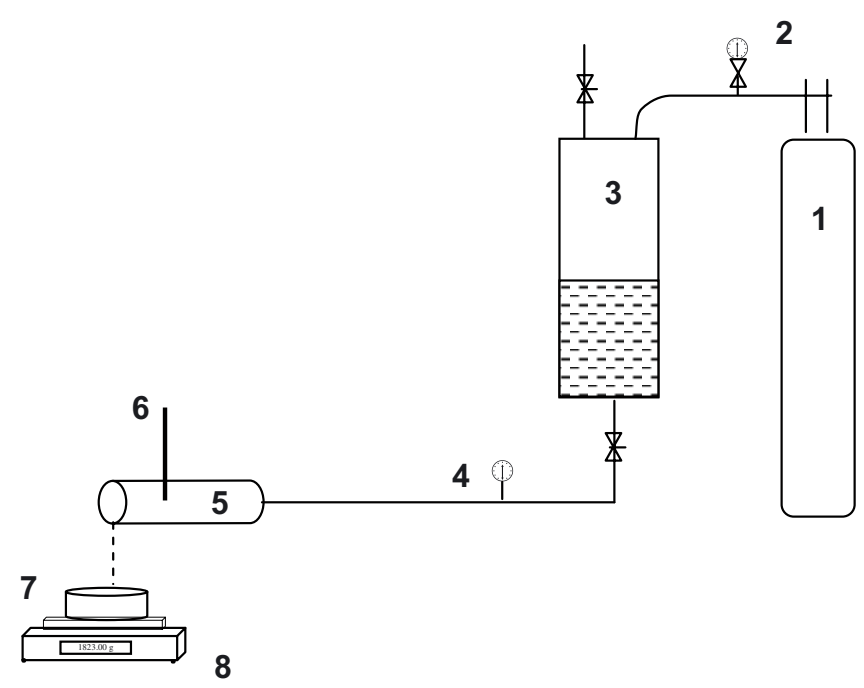

Figure 1. Schematic view of the testing bench used in the experiment (1): compressed nitrogen tank; (2): pressure regulator; (3): water reservoir; (4): pressure gauge; (5): olive tree branch; (6): TDP; (7): container; (8): balance.

flow density (between 0 and $t_{i}$ ) and the index $I\left(t_{i}\right)$. This relationship is expressed as

$$
\bar{u}=a I\left(t_{i}\right)+b
$$

where $a=C /\left(\alpha h_{0}\right)$ and $b=-1 / \alpha$ are constant coefficients. Simple linear relationships of the form of equation (8) between mean sap flow density and thermal index $I\left(t_{i}\right)$ will be experimentally calibrated. If we consider the heating phase, starting just when the current is switched on, the same reasoning holds, but equation (7) should be written as (see appendix)

$$
I\left(t_{i}\right)=\frac{1}{t_{i}} \ln \left(\frac{T_{e}-T_{0}}{T_{e}-T\left(t_{i}\right)}\right)
$$

where $T_{e}$ is the equilibrium temperature of the probe when the stationary regime is reached.

\subsection{Experimental setting}

An experiment was carried out to test the theory developed above and to calibrate a relationship between flow density and the thermal indices defined above. The basic idea of the method is to record, with a given time step, the temperature of the probe just after the heating current is switched on or switched off. A thermal dissipation probe (TDP) based on the original Granier's method was designed. This probe consists of a single needle with a heating element and a $T$-type thermocouple. Cold junction compensation is measured by a temperature sensor, NTC type (Negative Temperature Coefficient) protected by an isothermal shield.

A testing bench (Fig. 1) was used to carry out the experiment. The heating probe was inserted radially into an olive tree (Olea europaea $L$.) branch of about $5 \mathrm{~cm}$ diameter. The bark was removed near the probe so that the effective probe length $(2 \mathrm{~cm})$ was entirely located in the sapwood. Olive wood is classified as a diffuse porous xylem where vessels are distributed quite uniformly in the annual growth

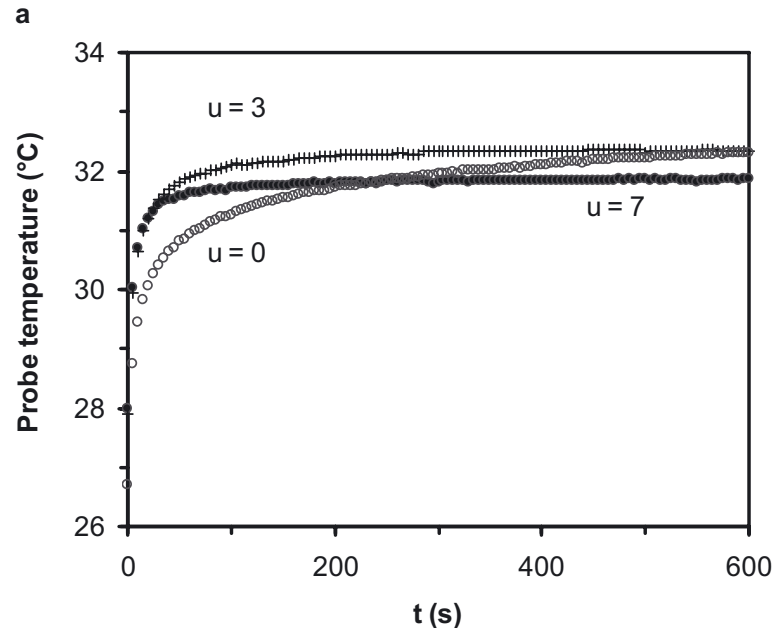

b

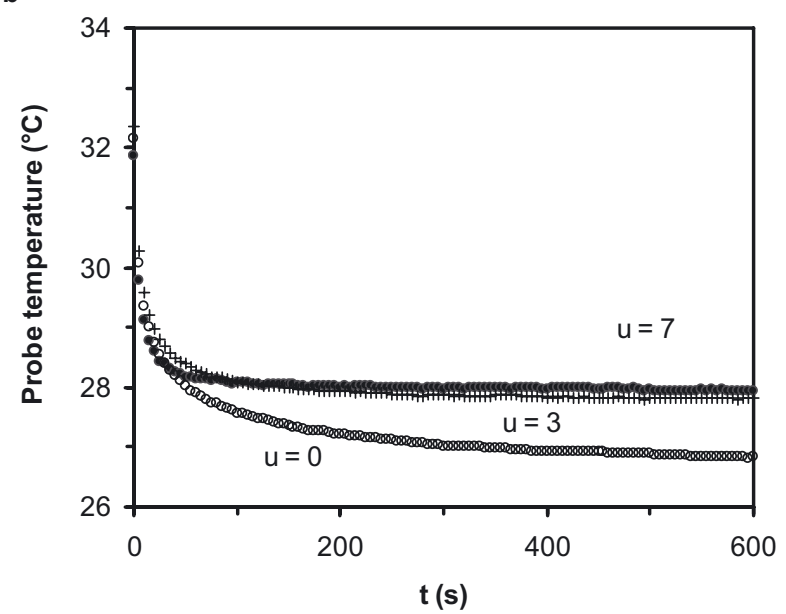

Figure 2. Kinetics of probe temperature for different values of flow density $(u)$ expressed in $\mathrm{L} \mathrm{h}^{-1} \mathrm{dm}^{-2}$ : (a) during the heating phase and (b) during the cooling phase. Ambient temperature was not constant during the measurements.

rings. Water was injected into the olive tree branch from a reservoir maintained under pressure by means of compressed nitrogen, the flow rate being set by means of a pressure regulator. The water flowing out of the branch is collected into a container and weighed by a digital balance. A data logger (Delta-T devices, Cambridge, UK) was used to control the heating and to record the signals from the thermocouples, thermistor and digital balance. A relatively high frequency of temperature measurement is adopted because the temperature variation is fairly rapid for high flow densities. Measurement sequences using $5 \mathrm{~s}$ time step are taken during a period of $10 \mathrm{~min}$ just after heating current is switched on or off. Measurements were made for twelve levels of flow density from 0 to $9 \mathrm{~L} \mathrm{~h}^{-1} \mathrm{dm}^{-2}$ covering the whole range of sap flow, as found in field experiments on olive trees.

Sapwood cross area was determined by injecting water containing red-dye safranin through the olive tree branch. As almost all the wood area was colored in fuchsia, we considered that the entire section was conductive. 


\section{RESULTS}

\subsection{Characteristics of the kinetics}

Figure 2 shows the variation of probe temperature during the heating phase and the cooling phase for different values of flow density. For $u=3 \mathrm{~L} \mathrm{dm}^{-2} \mathrm{~h}^{-1}$ and $u=7 \mathrm{~L} \mathrm{dm}^{-2} \mathrm{~h}^{-1}$ and for both heating and cooling periods, an equilibrium state is reached within $10 \mathrm{~min}$ and a plateau is clearly visible. The time needed for the kinetic to be completed decreases when flow density increases. For low densities no clear plateau can be noticed within the $10 \mathrm{~min}$. It has been found that the difference between probe temperature measured after 10 and $20 \mathrm{~min}$ of cooling does not exceed $2 \%$ of the temperature recorded at $10 \mathrm{~min}$ for a wide range of flow density. Once the kinetic is completed, temperature remains approximately constant, if there is no change in the environmental conditions. This temperature depends on the level of flow density. Equation (A.2) in the appendix shows that for a given heating power, the higher the flow density (and thus the heat exchange coefficient $h(u)$ ), the lower the equilibrium temperature $T_{e}$. During the cooling phase, the stationary temperature $T_{w}$ adjusts to ambient temperature variations (Fig. 2b).

In fact, the probe temperature was found not to fit the strict exponential variation with time that could be expected from the theory developed above assuming flow density to remain constant. In similar experiments involving nonstationary regimes, Benet et al. (1977) reported that the exponential decrease occurs only after a short period of time, during which the variation of temperature is faster than the exponential variation predicted by the theory, for a reason not completely elucidated yet. This behavior was verified in our experiment: a rapid cooling or heating is observed during a short period, and then the temperature variation slows down, the experimental points approximately fitting an exponential curve. The delay needed for the exponential variation to appear depends on flow density level. The duration of the exponential phase also varies with flow density. The theory developed above (Eq. (5)) demonstrates that under a constant flow density, and thus a constant heat exchange coefficient, $y(t)=\ln \left[\left(T_{0}-T_{w}\right) /\left(T(t)-T_{w}\right)\right]$ should be a linear function of time during the cooling phase ( $T_{w}$ being approximated by the probe temperature $10 \mathrm{~min}$ after switch off). As shown in Figure 3, where $y(t)$ is plotted against time, the strict exponential phase, which corresponds here to the linear part of the curve, is in fact delayed with respect to the switch off time. The delay before the linear phase is reached and the duration of this linear phase decrease with increasing flow density. It appears also from Figure 3 that the greater the flow density, the higher the slope of the line.

\subsection{Calibration and validation}

The experimental data set corresponding to several repetitions of measurement sequences of heating and cooling was split into two subsets, one (A) for calibration and the other (B) for validation. The consecutive cycles were alternatively put

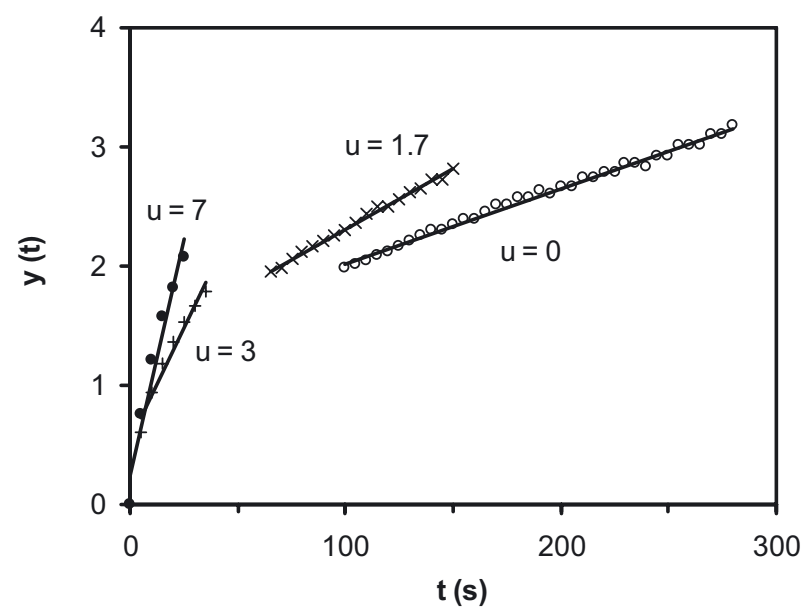

Figure 3. Linear part of function $y(t)$ defined by equation (5) versus time during the cooling phase of the probe for different levels of flow density $u\left(\mathrm{~L} \mathrm{~h}^{-1} \mathrm{dm}^{-2}\right)$.

Table I. Calibration equations $u=a I\left(t_{i}\right)+b$ between the flow density $u$ and the thermal index $I$ for $t_{i}$ ranging from $10 \mathrm{~s}$ to $60 \mathrm{~s} . R^{2}$ is the coefficient of determination of the regression line (17 and 13 observations respectively for heating and cooling modes).

\begin{tabular}{ccccccc}
\hline$t_{i}(\mathrm{~s})$ & \multicolumn{3}{c}{ Heating mode } & \multicolumn{3}{c}{ Cooling mode } \\
\hline & $a$ & $b$ & $R^{2}$ & $a$ & $b$ & $R^{2}$ \\
\hline 10 & -200 & 13.8 & 0.93 & 144 & -10.2 & 0.96 \\
20 & -435 & 10.6 & 0.90 & 180 & -8.7 & 0.96 \\
30 & -725 & 9.4 & 0.89 & 216 & -8.2 & 0.95 \\
40 & -1056 & 8.7 & 0.87 & 256 & -8.2 & 0.94 \\
50 & -1424 & 8.2 & 0.84 & 277 & -7.8 & 0.95 \\
60 & -1854 & 7.9 & 0.83 & 315 & -7.9 & 0.95 \\
\hline
\end{tabular}

in subset A and B. Thermal indices $I\left(t_{i}\right)$ were calculated for $t_{i}$ ranging from 10 to $60 \mathrm{~s}$ every $10 \mathrm{~s}, T_{w}$ and $T_{e}$ being taken at $t=600 \mathrm{~s}$. The temperature recorded after $10 \mathrm{~min}$ was used as an approximation of the probe temperature at equilibrium for both heating and cooling periods. Correlations between thermal indices $I\left(t_{i}\right)$ and gravimetrically measured water flow densities $\left(u_{m e}\right)$ were determined for both heating and cooling periods with data subset $\mathrm{A}$. The coefficients of the regression lines $u_{m e}=a I\left(t_{i}\right)+b$ with the corresponding coefficient of determination $\left(R^{2}\right)$ are given in Table I. First, it appears that the proposed thermal indices $I\left(t_{i}\right)$ are generally well correlated to measured water flow density with $R^{2}$ values in the range $0.83-0.96$. Second, the linear regressions are better for cooling mode than for heating mode. Third, the highest $R^{2}$ values are obtained for thermal indices $I\left(t_{i}\right)$ calculated at $t_{i}=10 \mathrm{~s}$, $20 \mathrm{~s}$ and $30 \mathrm{~s}$ during the cooling phase, the corresponding regression lines are shown in Figure 4. Beyond $t_{i}=60 \mathrm{~s}$, the $R^{2}$ values decrease substantially.

In the validation work, the coefficients of the linear regression $u=a I\left(t_{i}\right)+b$ given in Table I for the cooling phase were used to estimate the values of flow density $\left(u_{e s}\right)$ with the independent data subset B. In order to select the most appropriate value of $t_{i}$, the root mean square errors (RMSE) and the 


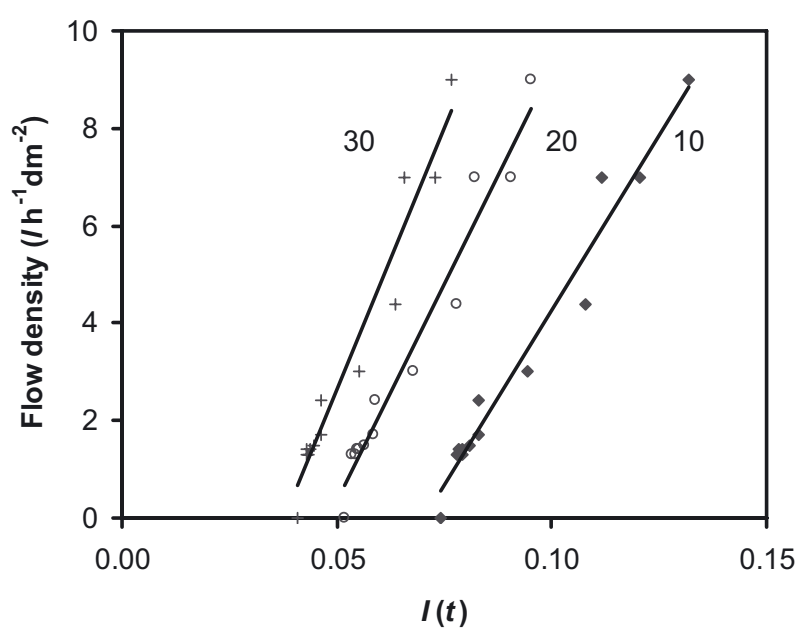

Figure 4. Regression lines of the gravimetrically measured water flow density $u_{\text {me }}$ versus $I\left(t_{i}\right)$ for $t_{i}=10,20$ and 30 s during the cooling phase (data set A).

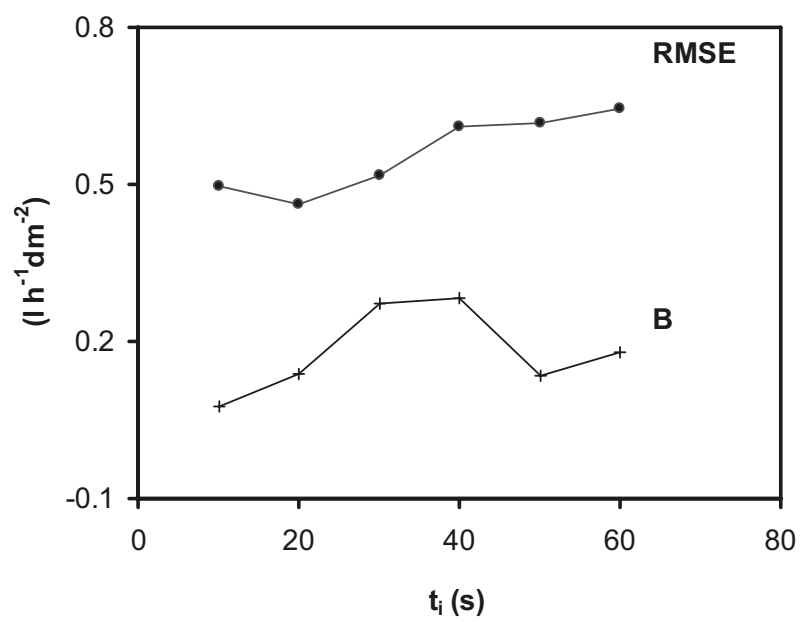

Figure 5. Root mean square errors (RMSE) and bias (B) between the gravimetrically measured water flow densities and values estimated by equation (8) using $I\left(t_{i}\right)$ in the cooling phase for $t_{i}$ ranging from $10 \mathrm{~s}$ to $60 \mathrm{~s}$.

bias (B) between $u_{e s}$ (estimated flow density) and $u_{m e}$ (gravimetrically measured flow density) were calculated for $t_{i}$ varying form 10 to $60 \mathrm{~s}$ (Fig. 5). The lowest RMSE is observed for $t_{i}=20 \mathrm{~s}$ with an acceptable bias, suggesting that it is the most appropriate time, at least for our experiment. Figure 6 compares the measured values of water flow density with those estimated using the regression equation corresponding to $t_{i}=20 \mathrm{~s}$. With a flow density in the range $0-7 \mathrm{~L} \mathrm{~h}^{-1} \mathrm{dm}^{-2}$ the RMSE value is $0.46 \mathrm{~L} \mathrm{~h}^{-1} \mathrm{dm}^{-2}$ and the bias is $0.14 \mathrm{~L} \mathrm{~h}^{-1}$ $\mathrm{dm}^{-2}$ which is rather satisfactory. For $t_{i}=10 \mathrm{~s}$ and $t_{i}=30 \mathrm{~s}$, RMSE values are also acceptable with respectively $0.50 \mathrm{~L} \mathrm{~h}^{-1}$ $\mathrm{dm}^{-2}$ and $0.52 \mathrm{~L} \mathrm{~h}^{-1} \mathrm{dm}^{-2}$. These results confirm the robustness of this new approach based upon the transient regime of a single probe, which can be a reliable method of sap flow measurement.

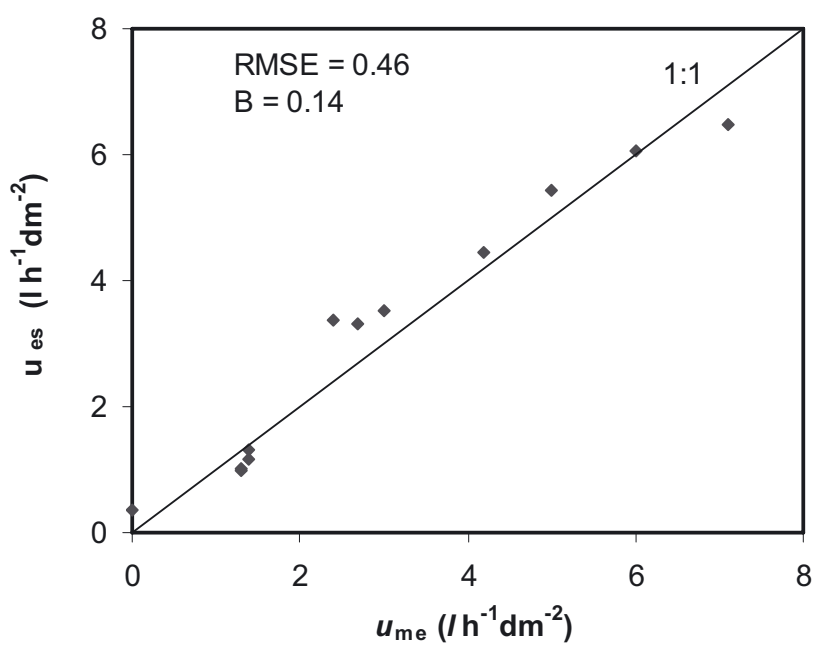

Figure 6. Gravimetrically measured water flow densities versus estimated values using $I\left(t_{i}\right)$ in the cooling phase with the intermediate time $t_{i}=20 \mathrm{~s}$ (data set B).

\section{DISCUSSION}

The techniques of sap flow measurement by thermal dissipation probe with constant heating (Granier, 1985) and cyclic heating (Do and Rocheteau, 2002b) have been revisited. It has been shown that the transient regime of the single probe occurring just after the heating current is switched off allows the definition of a thermal index well correlated to flow density. This index is expressed as a combination of three temperatures of the probe measured at three different times of the kinetic: initial temperature $T_{0}$, final temperature $T_{w}$ and an intermediate temperature $T\left(t_{i}\right)$ taken between $t_{i}=10 \mathrm{~s}$ and $30 \mathrm{~s}$. This thermal index is the basis of a transient approach of sap flow measurement by a single probe.

When compared to the previous methods, this approach appears to have several advantages. The first practical advantage is its simplicity, since only one probe is needed with the same complexity in electronic control as the cyclic method of Do and Rocheteau (2002b). Second, the problems linked to thermal gradients within the sapwood, when constant heating is used, are eliminated since the temperature is measured only at one location. Additionally, the method does not need, in principle, the determination of zero flow conditions, which are not easy to identify practically, and which are required in Granier's method (to eliminate the heating power $R I^{2}$ in the equations) as well as in Do and Rocheteau's one. Nevertheless, if the thermal index in zero flow conditions $I_{0}\left(t_{i}\right)$ is known, the calibration relationship (represented by equation (8)) simply transforms into $u=-b K$, where $K=\left[I\left(t_{i}\right) / I_{0}\left(t_{i}\right)\right]-1$ is a dimensionless index similar to the one used in Granier's method. However, this new relationship, in which the heat exchange coefficient at zero flow $\left(h_{0}\right)$ is eliminated, is not fundamentally different from equation (8) and apparently not more accurate. An inherent problem with the method of Do and Rocheteau (2001b) is the non-steady state nature of the sap flow during 
the measurement period. Indeed, in periods of rapid changing meteorological conditions (early morning, late afternoon or cloudy conditions), steady flow rarely happens. It is not clear how this difficulty is resolved in their method. This problem apparently does not exist in our approach. The theory shows that it is the mean flow between $t=0$ and the intermediate time $t_{i}$, which is taken into account (a period of time lower than $30 \mathrm{~s}$ in principle). The period after $t=t_{i}$, is necessary to allow for the probe to reach the wood temperature $T_{w}$ which should remain constant.

In our experiment, measurement periods were arbitrarily set to $10 \mathrm{~min}$ in order to approximate stationary wood temperature. For both cooling and heating modes, additional $10 \mathrm{~min}$ were used respectively for heating and cooling between consecutive measurement sequences. However, as the initial temperature $T_{0}$ involved in the thermal index for cooling mode is not necessarily at equilibrium, a shorter heating period could be adopted and would result in a faster cooling. The number of sap flow measurements per hour could therefore be increased. For the selection of cooling duration, a compromise should be found: if the cooling phase is too short, the probe will not approach the wood equilibrium temperature $\left(T_{w}\right)$; if it is too long, flow density and external conditions may change and $T_{w}$ will no longer be related to the initial phase $\left(t_{i}\right)$. Additional experiments will be carried out to confirm the possibility of shortening the measurement sequences and further field work will test the single probe transient method for measuring tree sap flow. It will be also relevant to check if the calibration relationships obtained with an olive tree branch are independent of the medium used, as for Granier's relationship.

\section{Appendix: Theoretical basis of the transient method in the heating phase}

When the heating phase is considered, starting when the Joule effect (through a resistance $R$ and a current intensity $I$ ) is switched on, the transient heat balance equation is written as

$$
C \frac{d T}{d t}+h(u)\left(T-T_{w}\right)=R I^{2}
$$

with the same symbols as those used in the main text. When the steady-state regime is reached, assuming all the heat produced by the probe to be exchanged with the wood, the equilibrium temperature of the probe is denoted by $T_{e}$ and equation (A.1) transforms into

$$
R I^{2}=h(u)\left(T_{e}-T_{w}\right)
$$

This means that equation (A.1) can be rewritten as

$$
C \frac{d T}{d t}+h(u)\left(T-T_{e}\right)=0 .
$$

The solution of which is

$$
T(t)-T_{e}=\left(T_{0}-T_{e}\right) \exp [-h(u) t / C]
$$

where $T_{0}$ is the temperature of the probe at $t=0$ and $T_{e}$ is the equilibrium temperature of the probe when the stationary regime is reached. Consequently, an equation very similar to equation (5) can be written as

$$
y(t)=\ln \left(\frac{T_{e}-T_{0}}{T_{e}-T(t)}\right)=\frac{h(u)}{C} t
$$

which leads to equation (9).

\section{List of symbols}

C heat capacity of the probe

$h$ exchange coefficient of the probe

$h_{0} \quad$ exchange coefficient of the probe at zero flow

$I \quad$ thermal index defined by equation (7) or (9)

$T(t)$ temperature of the probe at time $t$

$T_{\mathrm{e}} \quad$ temperature of the probe at equilibrium with constant heating

$t_{i} \quad$ intermediate time of the kinetic

$T_{0} \quad$ initial temperature of the probe when current is switched off (or on)

$\mathrm{T}_{w} \quad$ temperature of the wood

$u \quad$ sap flow density

$\alpha$ parameter involved in the exchange coefficient $h$ of the probe (Eq. (2))

$\beta \quad$ parameter involved in the exchange coefficient $h$ of the probe (Eq. (2))

\section{REFERENCES}

Andrade J.A., Meinzer F.C., Goldstein G., Holbrook N.M., Cavelier J., Jackson P., and Silvera K., 1998. Regulation of water flux through trunks, branches and leaves in trees of a lowland tropical forest. Oecologia 115: 463-471.

Benet J.C., Pomade P., and Hinzelin P., 1977. Mesure de la conductivité thermique des sols par sonde cylindrique en régime transitoire. Etude de l'influence du mode de mise en place de la sonde. Matér. constr. 10: $385-392$.

Braun P. and Schmid J., 1999. Sap flow measurements in grapevines (Vitis vinifera L.). 2. Granier measurements. Plant Soil 215: 47-55.

Cabibel B. and Do F., 1991. Mesures thermiques des flux de sève dans les troncs et les racines et fonctionnement hydrique des arbres. I. Analyse théorique des erreurs sur la mesure des flux et validation des mesures en présence de gradients thermiques extérieurs. Agronomie 11: 669-678.

Do F. and Rocheteau A., 2002a. A cyclic thermal dissipation system for measuring sap flow under high natural wood temperature gradients. I. Gradients and signals in the field. Tree Physiol. 22: 641-648.

Do F. and Rocheteau A., 2002b. A cyclic thermal dissipation system for measuring sap flow under high natural wood temperature gradients. II. Analysis in an artificial flow system. Tree Physiol. 22: 649-654.

Granier A., 1985. Une nouvelle méthode pour la mesure du flux de sève brute dans le tronc des arbres. Ann. Sci. For. 42: 193-200.

Granier A., 1987. Evaluation of transpiration in a Douglas-fir stand by means of sap flow measurements. Tree Physiol. 3: 309-320.

Lu P., Urban L., and Zaho P., 2004. Granier's Thermal dissipation probe (TDP) method for measuring sap flow in trees: theory and practice. Acta Bot. Sin. 46: 631-646.

Lüttschwager D. and Remus R., 2007. Radial distribution of sap flux density in trunks of a mature beech stand. Ann. For. Sci. 46: 431-438. 
Ma L., Lu P., Zhao P., Rao X., Cai X., and Zeng X., 2008. Diurnal, daily, seasonal and annual patterns of sap-flux-scaled transpiration from an Acacia mangium plantation in southern China. Ann. For. Sci. 65: 402.

Marshall D.C., 1958. Measurement of sap flow in conifers by heat transport. Plant Physiol. 6: 385-396.

Masmoudi M.M., Mahjoub I., Charfi-Masmoudi C., Abid-Karray J., and Ben Mechlia N., 2004. Mise au point d'un dispositif de mesure du flux de sève xylémique chez l'olivier. Rev. Rég. Arides ns: 242-251.

Meinzer F.C., Goldstein G., Franco A.C., Bustamente M., Igler E., Jackson P., Caldas L., and Rundel P.W., 1999. Atmospheric and hydraulic limitations on transpiration in Brazilian cerrado woody species. Funct. Ecol. 13: 273-282.

Oliveras I. and Llorens P., 2001. Medium-term sap flux monitoring in a Scots pine stand: analysis of the operability of the heat dissipation method for hydrological purposes. Tree Physiol. 21: 473-480.
Sevanto S., Nikinmaa E., Riikonen A., Daley M., Pettyjohn J.C., Mikkelsen T.N., Phillips N., and Holbrook N.M., 2008. Linking xylem diameter variation with sap flow measurements. Plant soil 305: $77-90$.

Valancogne C. and Granier A., 1991. Intérêt des méthodes thermiques de mesure du flux de sève pour l'étude du bilan hydrique des savannes. In Soil Water Balance in the Sudano-Sahelian Zone, Proceedings of the Niamey Workshop, IAHS Publ. 199: 387-400.

Wilson K.B., Hanson P.J., Mulholland P.J., Baldocch D.D., and Wullschleger S.D., 2001. A comparison of methods for determining forest evapotranspiration and its components: sap-flow, soil water budget, eddy covariance and catchment water balance. Agric. For. Meteorol. 106: 153-168.

Wullschleger S.D. and Hanson P.J., 2008. Sensitivity of canopy transpiration in an upland oak forest: evidence from a long-term field manipulation study. Glob. Chang. Biol. 12: 97-109. 